\title{
Silencing Dicer expression enhances cellular proliferative and invasive capacities in human tongue squamous cell carcinoma
}

\author{
SHUGUANG ZENG $^{1 *}$, JING YANG $^{1 *}$, JIANJIANG ZHAO ${ }^{1}$, QICAI LIU ${ }^{2}$, \\ MINGDENG RONG ${ }^{1}$, ZEHONG GUO $^{1}$ and WENFENG GAO ${ }^{1}$ \\ ${ }^{1}$ Stomatological Hospital of Guangdong Province Affiliated to Southern Medical University, Guangzhou, Guangdong 510280; \\ ${ }^{2}$ Department of Molecular Biology, Guangzhou Medical College, Guangzhou, Guangdong 510120, P.R. China
}

Received September 20, 2013; Accepted November 11, 2013

DOI: $10.3892 /$ or.2013.2903

\begin{abstract}
RNAs (miRNAs) are aberrantly expressed in cancer. An enzyme essential for miRNA processing is Dicer, whose expression is deregulated in diverse types of cancer and correlates with tumor progression. However, whether the regulation of Dicer expression affects tongue squamous cell carcinoma is unknown. In the present study, we investigated how silencing the expression of Dicer alters cell proliferation, cell cycle patterns, and cell migration and invasion in the Tca-8113 tongue squamous cell carcinoma cell line. Dicer expression levels were determined using quantitative PCR and western blot analysis in normal oral gingival epithelial cells and in two tongue squamous cell carcinoma lines, Tca-8113 and UM-1. Tca-8113 cells were transfected with Dicer siRNA or a negative control siRNA. Cell proliferation was determined using the MTT assay and the cell cycle was examined using flow cytometry. Cell migration and invasion changes were evaluated using wound-healing, adherence and Transwell assays. Dicer was expressed at lower levels in the tongue squamous cell carcinoma cell lines Tca-8113 and UM-1 compared to normal gingival epithelial cells, and less Dicer was expressed in UM-1 cells compared to Tca-8113 cells. Notably, Tca-8113 cells transfected with Dicer siRNA had significantly higher proliferative and invasive abilities than cells transfected with the negative control siRNA or nontransfected cells. Silencing Dicer may promote the progression of tongue squamous cell carcinoma. Dicer could serve a promising biomarker and a potential therapeutic target for tongue squamous cell carcinoma.
\end{abstract}

Correspondence to: Professor Shuguang Zeng or Professor Jianjiang Zhao, Guangdong Provincial Stomatological Hospital Affiliated to Southern Medical University, 366 Jiangnan Da Dao, Guangzhou 510280, P.R. China

E-mail: sunrisez@tom.com

E-mail: zjj2521@sina.com

*Contributed equally

Key words: Dicer, carcinoma, tongue, squamous cell, proliferation, invasion

\section{Introduction}

microRNAs (miRNAs) are small non-coding RNAs ( $22 \mathrm{nt})$ that regulate the expression of target genes by promoting RNA cleavage or inhibiting RNA translation. Dicer, a key member of the RNase III family, is an essential component of the miRNA-processing machinery in the cytoplasm in which the precursors of miRNAs are processed by Dicer into mature miRNAs (1). The abnormal expression of miRNAs is associated with the development and progression of cancer $(2,3)$. Dicer is aberrantly expressed in different types of cancer, and, being an upstream regulator of miRNAs, Dicer may affect the behavior of cancer by altering the expression spectrum of miRNAs.

The miRNA expression spectrum varies with cancer types, although in most types of cancer miRNAs are globally downregulated (4). However, whether the reduction in miRNA expression promotes cancer development or merely reflects the state of undifferentiated tumors is unclear. Markedly, tumor behaviors have been reported to be altered after silencing Dicer expression in vitro $(5,6)$, but little evidence is available regarding the expression patterns of Dicer in tongue squamous cell carcinoma or the influence of knocking down Dicer expression on this type of cancer. In the present study, we first compared Dicer protein and mRNA expression between 2 carcinoma cell lines, Tca-8113 $(7,8)$ and UM-1 $(8,9)$ and normal gingival epithelial cells. We found that Dicer protein levels were lower in the carcinoma cells than in normal cells, and that Dicer expression was the lowest in the highly aggressive UM-1 cancer cells. Thus, we knocked down the expression of Dicer in Tca-8113 cells using siRNA transfection in vitro to investigate how disrupting Dicer-dependent maturation of miRNAs affects cell proliferation, cell cycle patterns and cell migration and invasion to affect cancer development.

\section{Materials and methods}

Cell culture and siRNA transfection. Normal gingival epithelial cells were purchased from the American Type Culture Collection (ATCC; Manassas, VA, USA). Tca-8113 and UM-1 were kindly provided by Dr AnXun Wang (The First Hospital Affiliated with Sun Yat-Sen University, Guangzhou, China). Cells were grown in minimal essential medium containing 
dimethyl sulfoxide (DMSO); media used were MEM for normal gingival epithelial cells, RPMI-1640 for Tca-8113 cells, and DMEM for UM-1 cells, all supplemented with $10 \%$ fetal bovine serum (FBS; Gibco). Dicer siRNA and negativecontrol siRNA were synthesized by Shanghai Jima Co. (Shanghai, China). The target sequences for Dicer siRNA were: 5'-GCCAAGGAAAUCAGCUAAATT-3' and 5'-UUUAGCU GAUUUCCUUGGCTT-3'. The negative-control siRNA sequences were: 5'-UUCUCCGAACGUGUCACGUTT-3' and 5'-ACGUGACACGUUCGGAGAATT-3'.

One day before transfection, Tca- 8113 cells were re-plated in 6-well plates to obtain $50-80 \%$ confluence on the day of transfection. A complex of siRNAs (100 nM) and $5 \mu \mathrm{l}$ Lipofectamine RNAiMAX reagent (Invitrogen) was added into each well according to the manufacturer's instructions. Transfection efficiency was estimated $6 \mathrm{~h}$ later using fluorescence microscopy. Transfections were performed in triplicate for each treatment.

Real-time quantitative PCR analysis. Total RNA was extracted using TRIzol reagent (following the manufacturer's instructions; Invitrogen) from normal gingival epithelial cells, Tca- 8113 cells, UM-1 cells and 3 groups of Tca- 8113 cells: cells transfected with Dicer-siRNA and negative-control siRNA and non-transfected cells. From the total RNA, cDNAs were synthesized using the Takara PrimeScript RT reagent (Perfect Real-Time kit) under reaction systems for $500 \mathrm{ng}$ and $10 \mu \mathrm{l}$. The qPCR primer sequences used in the study were: Dicer forward primer, 5'-TGTGGGGAGAGGGCTGCTCA-3' and reverse primer, 5'-GGCACAGGGCCTTTTCCCGA-3'; GAPDH forward primer, 5'-AAGGTGAAGGTCGGAGTCA AC-3' and reverse primer, 5'-GGGGTCATTGATGGCAACA ATA-3'.

GAPDH was used as an internal control. PCR was performed in triplicate in a total reaction volume of $20 \mu \mathrm{l}$, which included $10 \mu \mathrm{l}$ of the $2 \mathrm{X}$ reaction mix, $0.4 \mu \mathrm{l}$ of 50X Rox, $3 \mu \mathrm{l}$ of primer $(2 \mu \mathrm{M}), 5.6 \mu \mathrm{l}$ of water and $1 \mu \mathrm{l}$ of cDNA, with SYBR Premix Ex Taq kit. The PCR protocol used involved denaturation for $30 \mathrm{sec}$ at $95^{\circ} \mathrm{C}$, followed by amplification for 40 cycles, each cycle consisting of $5 \mathrm{sec}$ at $95^{\circ} \mathrm{C}, 30 \mathrm{sec}$ at $56^{\circ} \mathrm{C}$ and $30 \mathrm{sec}$ at $72^{\circ} \mathrm{C}$. The median in each triplicate was used to calculate the relative Dicer expression level using the compara-

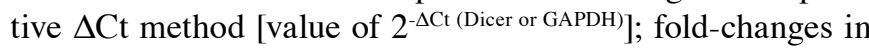
expression were calculated using $2^{-\Delta \Delta \mathrm{Ct}}$.

Western blotting. Total proteins were extracted from normal gingival epithelial cells, Tca-8113 cells, UM-1 cells and the 3 groups of Tca-8113 cells (transfected with Dicer-siRNA and negative-control siRNA and non-transfected) after transfection for 24 and $48 \mathrm{~h}$. Cells were washed thrice with phosphatebuffered saline (PBS) before being lysed on ice in $50 \mu \mathrm{l} 1 \mathrm{X}$ extraction buffer prepared fresh. Following centrifugation for $10 \mathrm{~min}$, supernatants were collected and aliquots were withdrawn for detecting proteins using Micro $\mathrm{BCA}^{\mathrm{TM}}$ protein assay reagent kit according to the manufacturer's instructions. SDS-PAGE (10\% gels) was used to resolve proteins (40 $\mu \mathrm{g} / \mathrm{lane})$, which were transferred electrophoretically for $2 \mathrm{~h}$ to polyvinylidene difluoride membranes. Membranes were blocked for $1 \mathrm{~h}$ in PBS-Tween $(0.1 \%)$ containing 5\% non-fat milk, probed overnight at $4^{\circ} \mathrm{C}$ with primary antibodies (rabbit anti-human Dicer, 1:500; Sigma, St. Louis, MO, USA), and then incubated for $1 \mathrm{~h}$ with secondary antibodies (goat antirabbit IgG, 1:1,000; Wuhan Boster Biological Technology, Ltd., Wuhan, China) for visualizing protein bands using enhanced chemiluminescence. Results shown are representative from experiments repeated at least twice.

Immunohistochemical staining. Dicer was labeled in normal gingival epithelial cells, Tca-8113 and UM-1 cells that had reached $80 \%$ confluence. Labeling with anti-human Dicer (Sigma) and secondary anti-rabbit IgG (Wuhan Boster Biological Technology) was according to the manufacturer's protocols using the SV-002 2-step-method Immunohistochemistry kit.

MTT assay. Cells were plated in 96-well plates at a density of 2,000 cells/well in $100 \mu \mathrm{l}$ of culture medium and maintained until they adhered before carrying out transfections. After transfection with siRNAs at $37^{\circ} \mathrm{C}$ for $4 \mathrm{~h}$, cells were incubated with $20 \mu \mathrm{l}$ of MTT solution $(5 \mathrm{mg} / \mathrm{ml})$ for 24 and $48 \mathrm{~h}$. At the end of the incubation, media were aspirated, $200 \mu 1$ of DMSO was added to each well and the absorbance at $570 \mathrm{~nm}$ was measured. Mean absorbance from 3 replicate wells was determined, and cell viability was defined as (absorbance of treated cells)/(absorbance of control cells).

Cell cycle analysis. The 3 groups of cells were harvested $24 \mathrm{~h}$ after transfection with siRNA; cells were fixed overnight with $70 \%$ cold ethanol, washed twice with cold PBS, and then incubated in RNase A $(10 \mathrm{mg} / \mathrm{ml})$ for $1 \mathrm{~h}$ at $37^{\circ} \mathrm{C}$. Propidium iodide (PI) $100 \mu \mathrm{g} / \mathrm{ml}$ was added and mixed for 30 min and then the cells were used for flow cytometric analysis.

Cell adhesion test, Erasion Trace test and Transwell cellinvasive assay. Matrigel (50 $\mu \mathrm{l})$ diluted with RPMI-1640 (1:5) was added to 96-well plates and maintained at $4^{\circ} \mathrm{C}$ overnight. The next day, plates were sealed at $37^{\circ} \mathrm{C}$ for $1 \mathrm{~h}$ after replacing the fluid in the wells with $100 \mu 1$ of RPMI-1640 containing $1 \%$ BSA. Experimental and control cells were plated without serum in the wells and incubated for $1 \mathrm{~h}$, and the cells that did not adhere to the Matrigel were washed off using PBS. Next, $20 \mu 1$ of MTT with $100 \mu 1$ of RPMI-1640 was added to each well and incubated for $4 \mathrm{~h}$. At the end of the incubation, the solution was aspirated, $200 \mu 1$ of DMSO was added to each well, and the absorbance at $570 \mathrm{~nm}$ was measured; mean absorbance was determined from 3 replicate wells.

When attached, transfected cells were $80 \%$ confluent in 24-well plates, the culture medium was replaced with basic medium, and a $100-\mu 1$ pipette tip was used to scrape cells in the middle of the wells. The scraped cells were washed off using PBS and the plates were placed in a $37^{\circ} \mathrm{C}$ incubator for $48 \mathrm{~h}$. Distances migrated by cells across the middle of the wells were calculated by examining cells from 5 fields selected randomly under the microscope.

To the top chamber of a Transwell chamber, $30 \mu 1$ of Matrigel diluted with RPMI-1640 (1:3) was added and maintained at $37^{\circ} \mathrm{C}$ and allowed to polymerize. Transfected cells were trypsinized and adjusted to a concentration of $5 \times 10^{5}$ cells $/ \mathrm{ml}$ in RPMI-1640, and $200 \mu \mathrm{l}$ of the resuspended cell solution was added to the top chamber above the Matrigel. The bottom chamber was filled with $600 \mu 1$ of RPMI-1640 containing $10 \%$ serum. The Transwell plates were incubated 

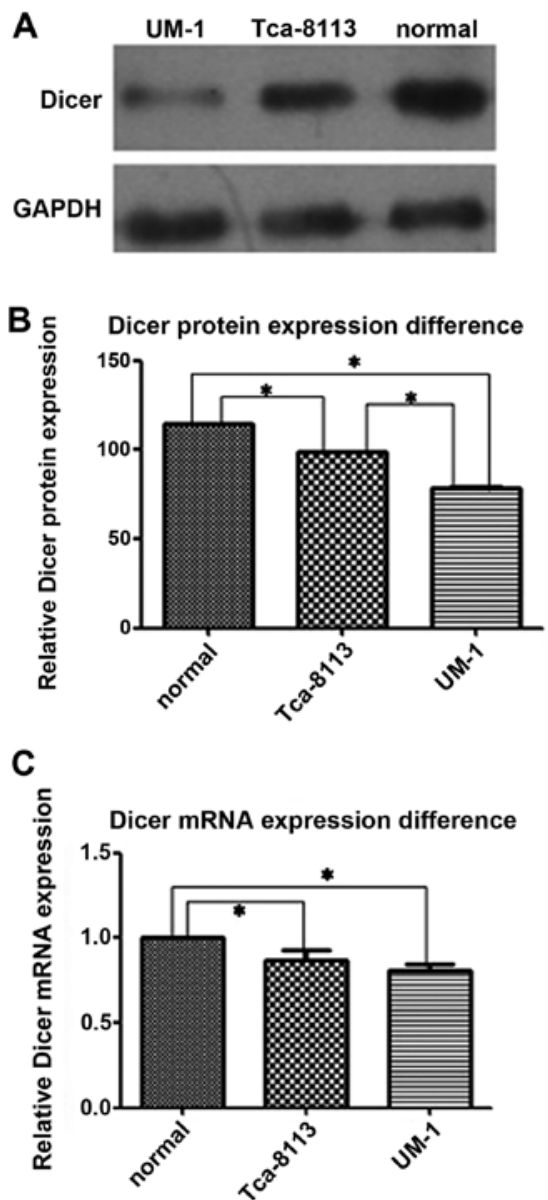

Figure 1. Dicer expression measured using western blotting and real-time qPCR. (A) Western blots showing the relative levels of Dicer staining in 3 types of cells; the band intensities are compared to GAPDH, which was used as a loading control. (B) Quantification of western blot staining showed that the protein level of Dicer was lower in Tca-8113 and UM-1 cells than in normal gingival epithelial cells, and that less Dicer protein was present in the high-grade cancer cell line UM-1 than in the lower-grade cell line Tca-8113 $\left({ }^{*} \mathrm{P}<0.05\right)$. (C) Real-time qPCR showed that Dicer mRNA expression in normal gingival epithelial cells was higher than in the 2 tongue squamous cell carcinoma cell lines ( $\mathrm{P}<0.05)$, whereas Dicer mRNA levels in Tca-8113 and UM-1 cells were not significantly different.

at $37^{\circ} \mathrm{C}$ for $24 \mathrm{~h}$ and then the top chamber was removed and the Matrigel with unmigrated cells was gently scraped with a wet cotton swab. Cells were stained with $0.1 \%$ crystal violet for $5 \mathrm{~min}$, washed with PBS to remove excess stain, and the average number of cells per field that had migrated was quantified under the microscope. The assay was repeated using the Transwell chamber without Matrigel.

Statistical analysis. SPSS-13.0 software, variance analysis, and 2 independent t-tests were used to analyze the differences in Dicer expression between the 3 types of cells and to evaluate the biological responses in treated and control cells.

\section{Results}

Dicer is downregulated in tongue squamous cell carcinoma cell lines. The relative expression levels of Dicer mRNA and protein were evaluated in normal oral gingival epithelial cells and 2 tongue squamous cell carcinoma cell lines, Tca- 8113

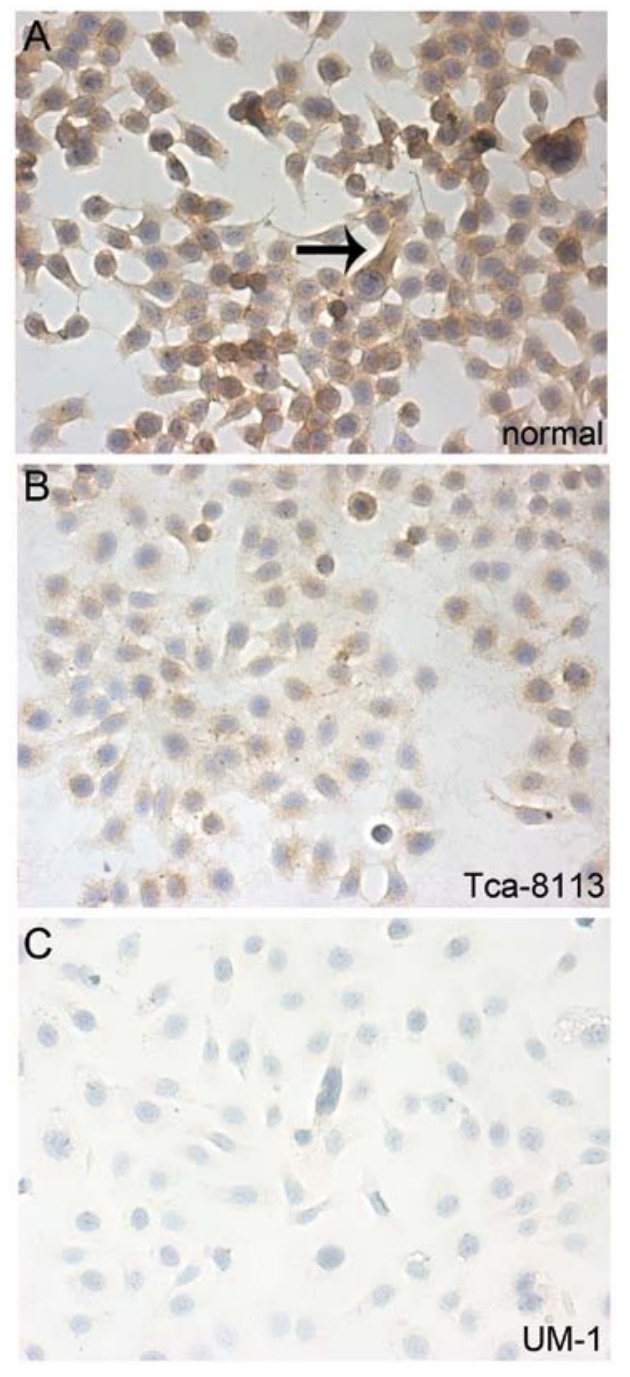

Figure 2. Dicer is expressed at higher levels in the normal gingival epithelial cells than in Tca-8113 and UM-1 cells. Dicer was mainly detected in the cytoplasm (arrow) by the immunohistochemical staining. (A) Dicer protein was strongly expressed in the cytoplasm of the normal gingival epithelial cells, whereas the expression was weaker in Tca-8113 cells (B) and the weakest in UM-1 cells (C). Original magnification, $\mathrm{x} 400$.

and UM-1 cells (Figs. 1 and 2). Real-time qPCR showed that more Dicer mRNA was present in normal oral gingival cells than in Tca-8113 cells $(\mathrm{P}=0.020)$ and $\mathrm{UM}-1$ cells $(\mathrm{P}=0.002)$. Western blotting showed that the amount of Dicer protein was significantly less in the 2 tongue squamous cell lines than in normal gingival epithelial cells $(\mathrm{P}=0.000$ for both Tca- 8113 and UM-1 cells). Dicer mRNA levels in Tca-8113 and UM-1 were not significantly different $(\mathrm{P}=0.166)$, but the amount of Dicer protein in the highly aggressive carcinoma cell line $\mathrm{UM}-1$ was less than in Tca- 8113 cells $(\mathrm{P}=0.000)$. Lastly, these results were further confirmed by the immunohistochemical staining for Dicer in the cells.

RNAi knocks down Dicer expression in Tca-8113 cells. FAM-labeled siRNAs were transfected into Tca- 8113 cells and $6 \mathrm{~h}$ later the transfection efficiency was determined using fluorescence microscopy. Approximately $80 \%$ of the transfected cells emitted green fluorescence upon excitation. Total cellular RNAs from Dicer siRNA-transfected cells and control cells 

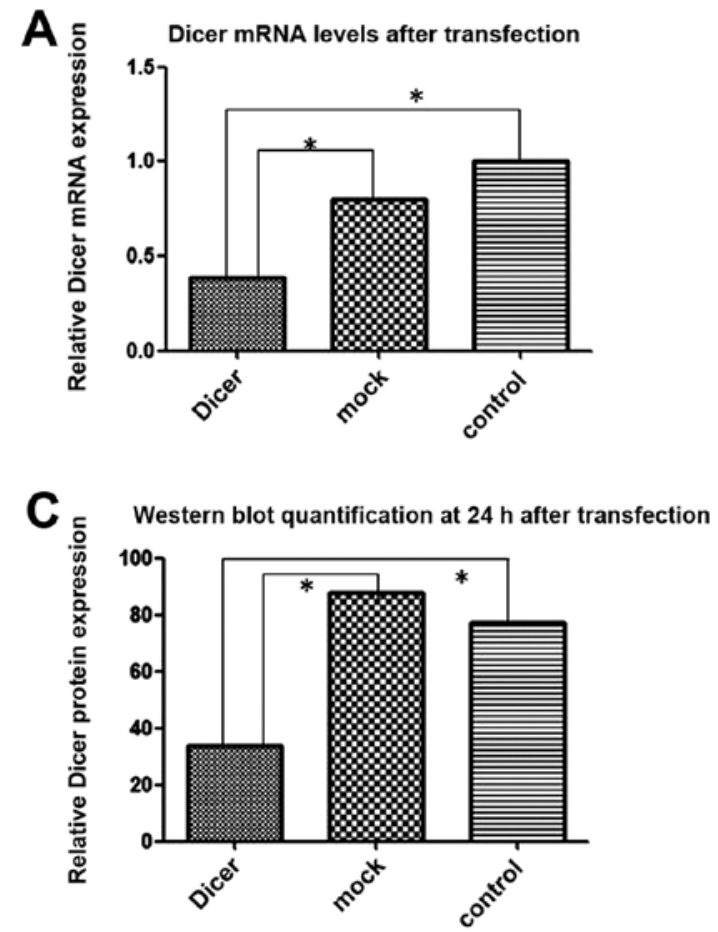
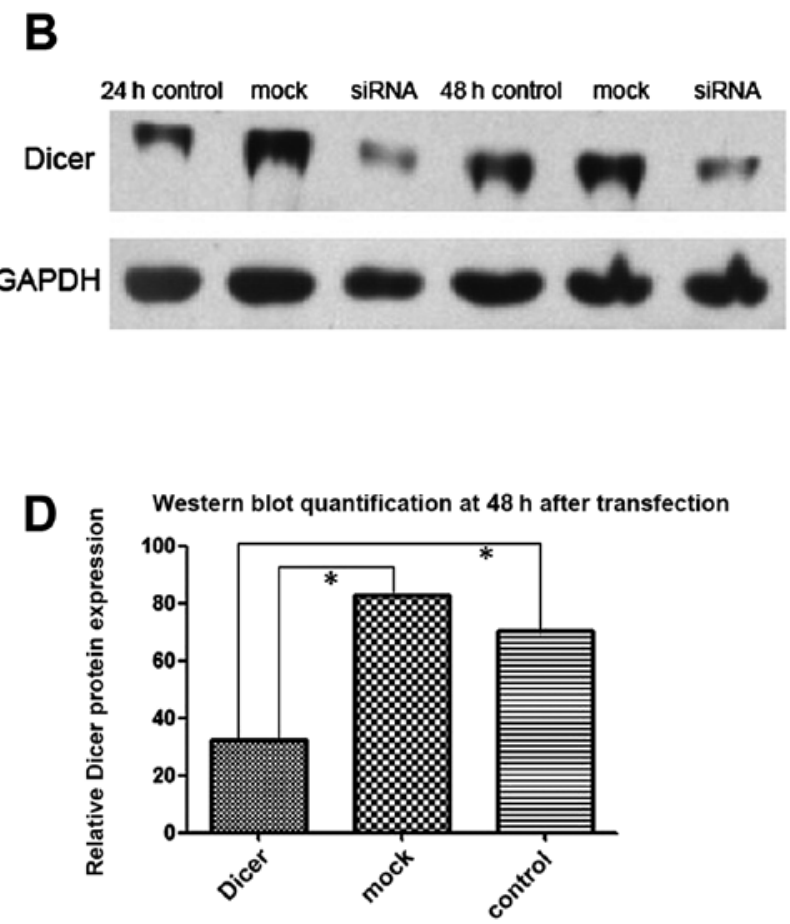

Figure 3. Dicer-siRNA reduces Dicer mRNA and protein expression in Tca-8113 cells. (A) Detection of Dicer and GAPDH mRNA using real-time qPCR in Tca-8113 cells $24 \mathrm{~h}$ after transfection showed a significant reduction of Dicer mRNA in cells transfected with Dicer-siRNA compared to negative-control siRNA-transfected cells and non-transfected cells ("P<0.05). (B) Western blot analysis showing Dicer protein for a comparison of band intensities (with GAPDH as a loading control) at 24 and $48 \mathrm{~h}$ after siRNA transfection. (C and D) Quantification of Dicer protein band intensities on western blots showed that Dicer was significantly downregulated in the experimental group (Dicer-siRNA) compared to the control groups ("P<0.05) at 24 and $48 \mathrm{~h}$ after transfection. Dicer, Tca-8113 cells transfected with Dicer-siRNA; mock, Tca-8113 cells transfected with negative-control siRNA; control, non-transfected Tca- 8113 cells; ${ }^{*} \mathrm{P}<0.05$.
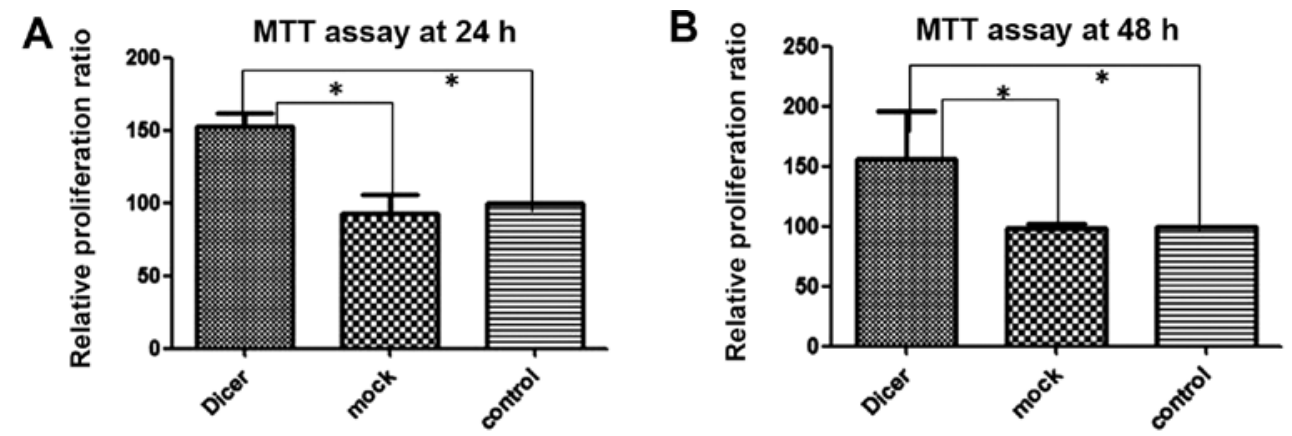

Figure 4. Knockdown of Dicer expression increases proliferation of Tca- 8113 cells. Cell viability was examined using the MTT assay. Our results showed that greater numbers of viable cells, representing increased proliferative ability, were detected at (A) $24 \mathrm{~h}$ after transfection $(\mathrm{P}=0.000)$ and $(\mathrm{B}) 48 \mathrm{~h}$ after transfection $(\mathrm{P}=0.011)$. Dicer, Tca-8113 cells transfected with Dicer-siRNA; mock, Tca-8113 cells transfected with negative-control siRNA; control, non-transfected Tca-8113 cells; ${ }^{\mathrm{P}}<0.05$.

(negative-control-siRNA transfected and non-transfected) were extracted $24 \mathrm{~h}$ after transfection. RT-PCR showed that the level of Dicer mRNA in the Dicer-siRNA group was significantly lower than in the 2 control groups $(\mathrm{P}=0.000)$. Similarly, western blotting demonstrated that the Dicer protein amount was substantially lower in the Dicer-siRNA group than in the 2 control groups at 24 and $48 \mathrm{~h}$ after transfection (Fig. 3).

Silencing Dicer promotes cell proliferation and cell cycle. To investigate the impact of Dicer on the proliferative capacity of cells, we measured the effects of Dicer-siRNA transfection on cell proliferation using the MTT assay and on cell cycle progression by using flow cytometry following PI staining. The
MTT assay (Fig. 4) demonstrated that transfection with DicersiRNA increased Tca- 8113 cell proliferation at 24 and $48 \mathrm{~h}$ after transfection relative to the 2 controls (negative-control siRNA transfected and non-transfected cells). Moreover, cell cycle analysis revealed that in Tca- 8113 cells transfected with Dicer-siRNA, the rate of $\mathrm{S}+\mathrm{G} 2$ phases increased sharply accompanied by a reduction in G1 phase (Fig. 5; Table I), indicating that the depletion of Dicer accelerated the cell cycle (to $\mathrm{S}$ and $\mathrm{G} 2$ phases).

Silencing Dicer expression increases cell migration and invasion. To examine how silencing Dicer expression affects the migratory and invasive abilities of Tca- 8113 cells, we quantified 

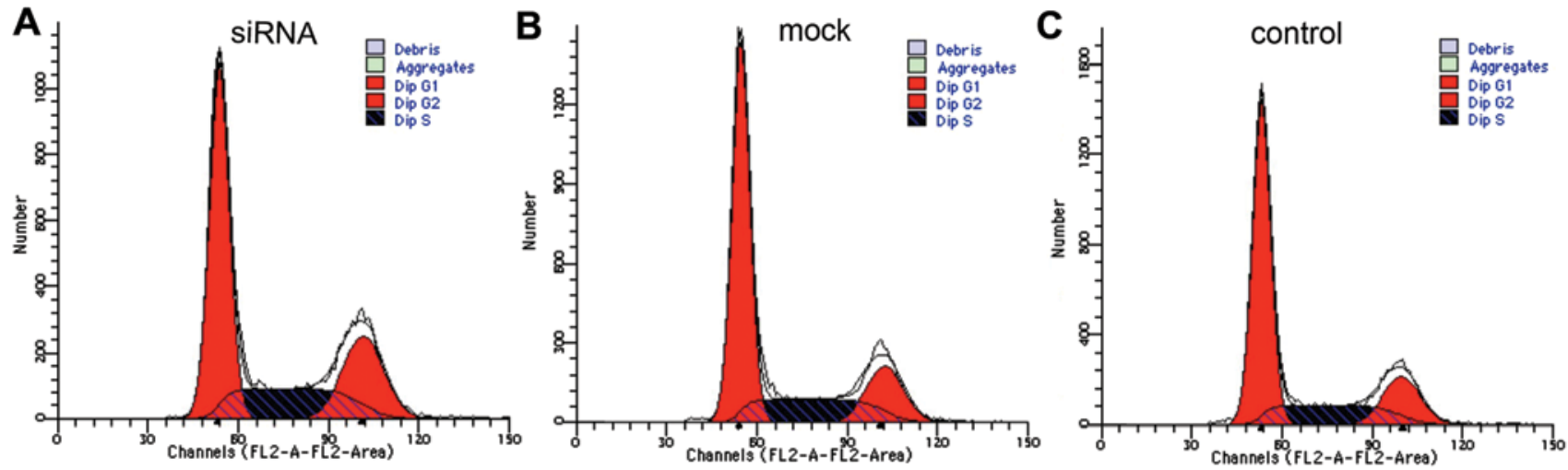

Figure 5. Silencing Dicer expression alter cell cycle distribution. Cell cycle analysis using flow cytometry demonstrated that Dicer downregulation increased the proliferative capacity of Tca-8113 cells. Cells in S+G2 phases made up 48.47,39.58 and 38.73\%, and cells in the G1 phase made up 51.42, 60.20 and $61.23 \%$, in Tca-8113 cells transfected with Dicer-siRNA, negative-control siRNA, and non-transfected cells, respectively. Thus, the cell cycle in the experimental group (Dicer-siRNA) had advanced to S phase. (A) siRNA, Tca-8113 cells transfected with Dicer-siRNA; (B) mock, Tca-8113 cells transfected with negative siRNA; (C) control, non-transfected Tca-8113 cells.
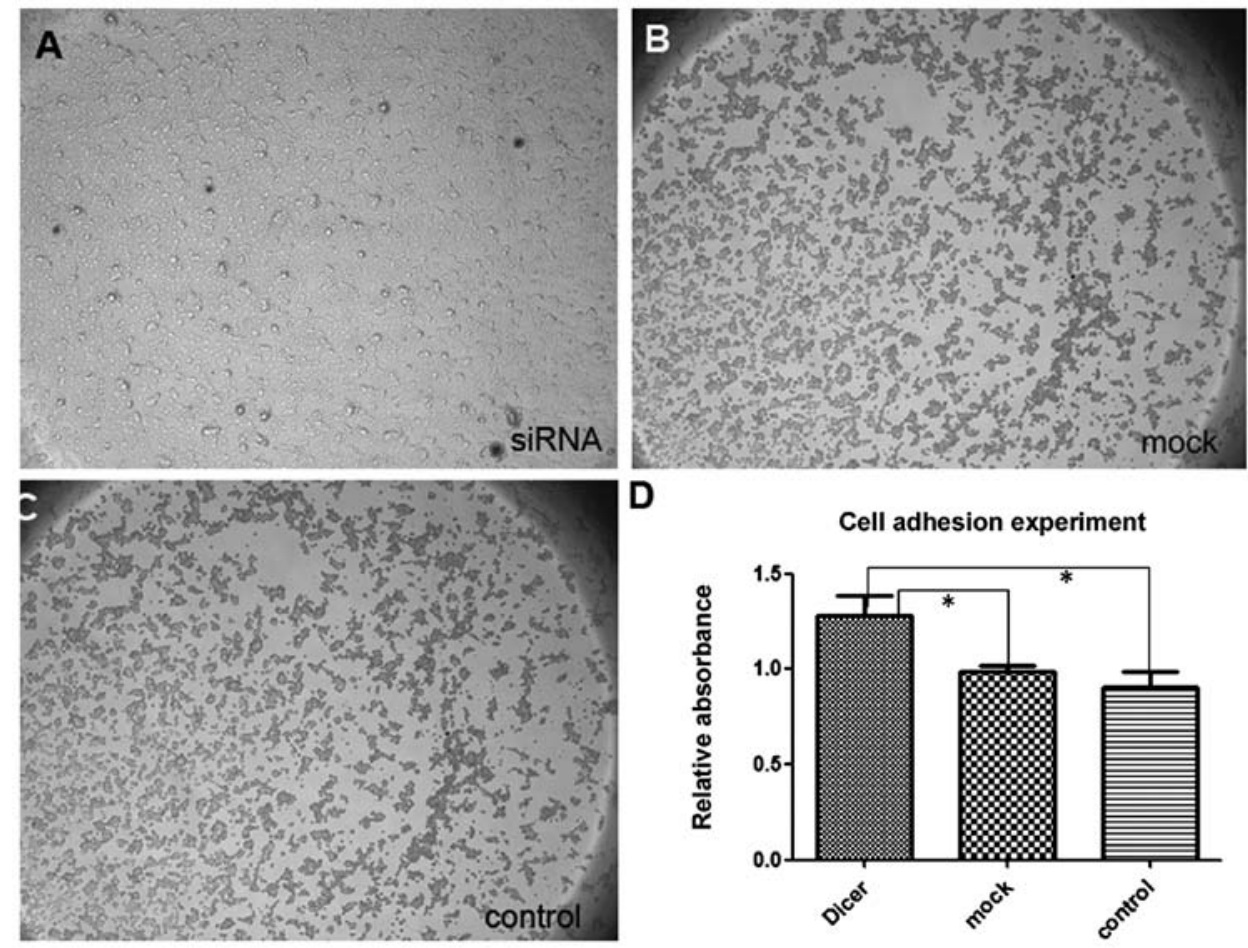

Figure 6. Knockdown of Dicer increases Matrigel adhesion of Tca-8113 cells. Knockdown of Dicer expression increased the number of cells attached to the Matrigel. (A) siRNA, Tca-8113 cells transfected with Dicer-siRNA; (B) mock, Tca-8113 cells transfected with negative-control siRNA; (C) control, nontransfected Tca-8113 cells. Original magnification, x100. (D) MTT assay results showing the increase in absorbance at $570 \mathrm{~nm}$ (which is positively correlated with the number of cells) in the experimental group compared to the mock and control groups; ${ }^{\mathrm{P}}<0.05$.

Table I. Cell cycle distribution.

\begin{tabular}{lcccc}
\hline $\begin{array}{l}\text { Cell } \\
\text { cycle }\end{array}$ & Control & Mock & siRNA & P-value \\
\hline G1 & $61.23 \pm 0.15$ & $60.20 \pm 0.04$ & $51.42 \pm 0.23$ & $0.000 / 0.001$ \\
S+G2 & $38.73 \pm 0.21$ & $39.58 \pm 0.35$ & $48.47 \pm 0.07$ & $0.004 / 0.014$ \\
\hline
\end{tabular}

G1 (siRNA vs. control, $\mathrm{P}=0.000$; siRNA vs. mock, $\mathrm{P}=0.001$ ); $\mathrm{S}+\mathrm{G} 2$ (siRNA vs. control, $\mathrm{P}=0.004$; siRNA vs. mock, $\mathrm{P}=0.014$ ). cell migration using cell adhesion and Erasion Trace tests and the Transwell cell-invasive assay. Compared to the control cells, more cells that had been transfected with Dicer-siRNA adhered to the Matrigel, migrated through the Transwell membrane and covered the erased trace better. Absorbance at $570 \mathrm{~nm}$ in the Matrigel adhesion test for Dicer-siRNA cells was $1.277 \pm 0.109$ compared to $0.990 \pm 0.026$ and $0.905 \pm 0.079$ for negative-control siRNA cells and non-transfected cells, respectively $(\mathrm{P}=0.011$, $\mathrm{P}=0.009$; Fig. 6). Similarly, Dicer-siRNA cells migrated more and covered the erased trace to $273.42 \pm 18.95 \mu \mathrm{m}$ compared to $175.76 \pm 5.81 \mu \mathrm{m}$ and $178.14 \pm 5.01 \mu \mathrm{m}$ for cells transfected 

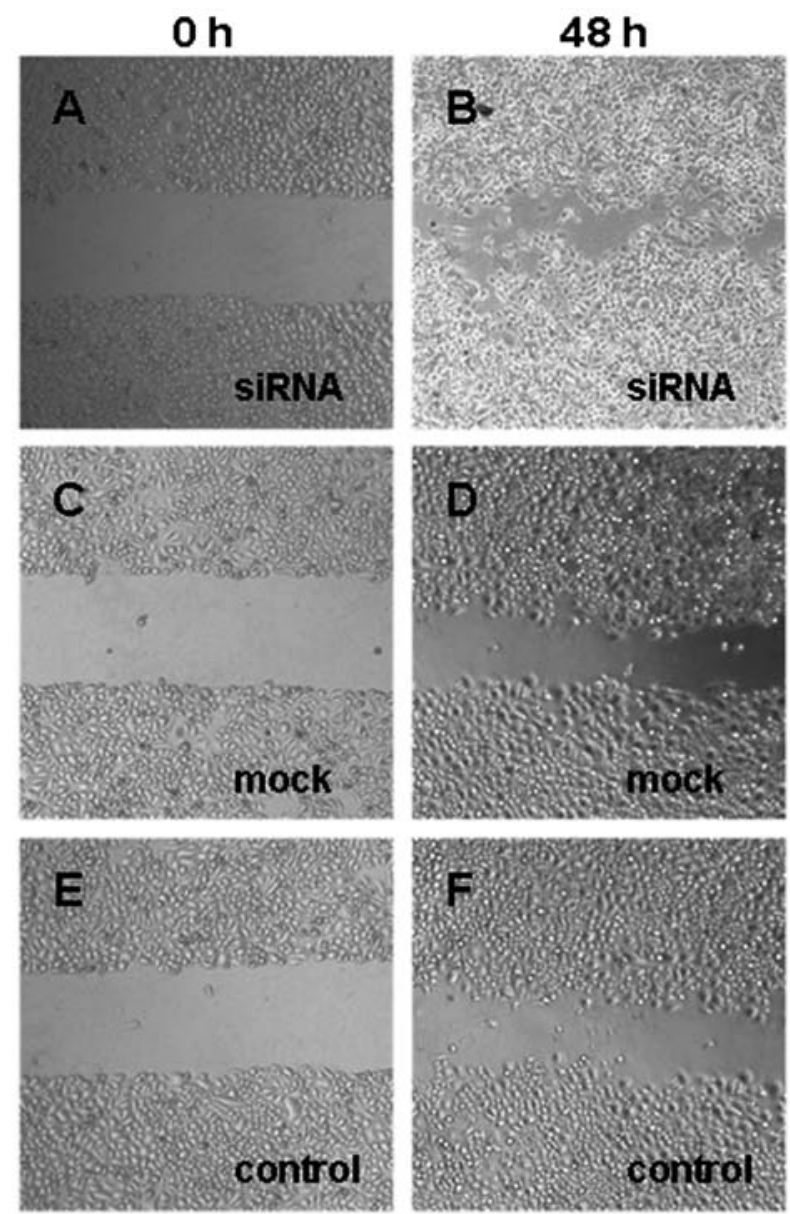

Figure 7. Silencing Dicer expression increases migration of Tca-8113 cells in Erasion Trace tests. Dicer-depleted cells migrated significantly farther than control cells $48 \mathrm{~h}$ after transfection. (A, C and E) Microscopic images for the 3 groups at $0 \mathrm{~h}$ in the Erasion Trace test. The distances between the cells are the same in the 3 groups. (B, D and F) Images captured $48 \mathrm{~h}$ after starting the Erasion Trace test. The distance between cells was significantly reduced in the experimental group compared to the control groups. (A and B) siRNA, Tca-8113 cells transfected with Dicer-siRNA; (C and D) mock, Tca-8113 cells transfected with negative-control siRNA; (E and F) control, non-transfected Tca-8113 cells. Original magnification, $\mathrm{x} 100$.

with the negative-control siRNA and non-transfected cells, respectively ( $\mathrm{P}=0.000, \mathrm{P}=0.000$; Figs. 7 and $9 \mathrm{~A})$. Furthermore, Dicer-siRNA transfection increased Transwell migration to $167.20 \pm 6.38$ cells from $92.40 \pm 13.22$ cells and $91.60 \pm 11.61$ cells in negative-control siRNA cells and non-transfected cells, respectively ( $\mathrm{P}=0.000, \mathrm{P}=0.000$; Figs. 8 and $9 \mathrm{~B})$. Therefore, transfecting Dicer-siRNA into Tca-8113 cells increased cell migration and invasion in vitro to levels significantly greater than in cells transfected with the negative-control siRNA and in non-transfected cells. Collectively, our results suggest that knocking down Dicer expression in Tca-8113 cells increases the invasive and proliferative capacities of these cells.

\section{Discussion}

Dicer, a member of RNase III family essential for miRNA processing, is known to be associated with cancer. Dicer is upregulated in prostate, colorectal cancer and lung squamous cell carcinoma $(1,10-14)$, and is downregulated in hepatocellular carcinoma, gastric, breast and ovarian cancer (15-18).
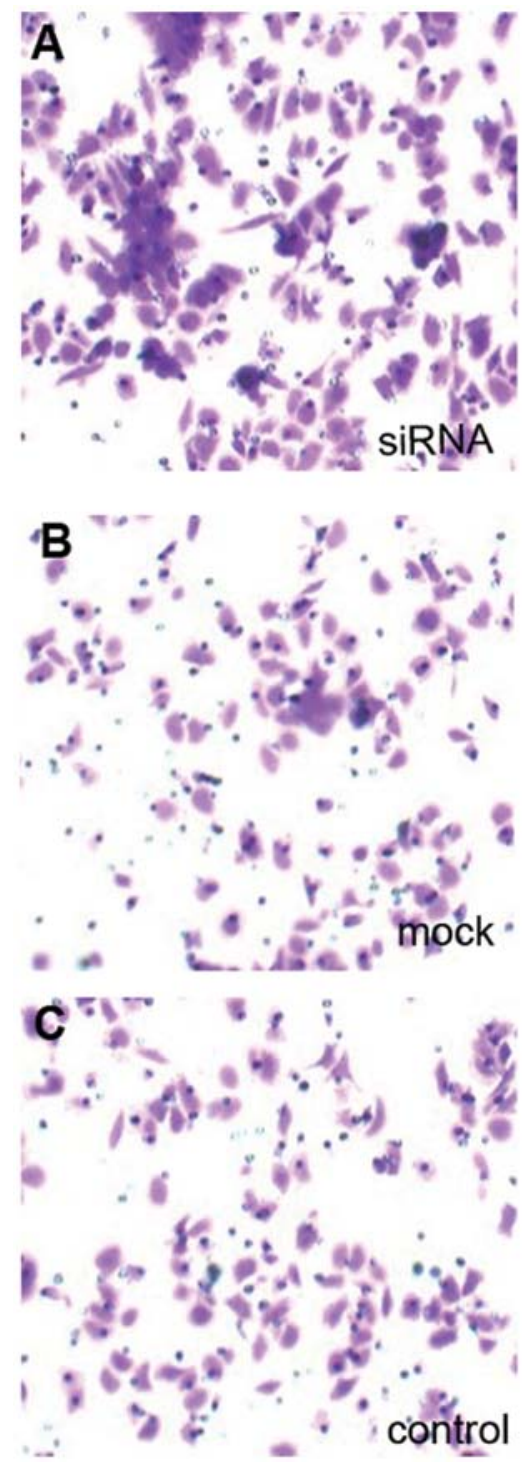

Figure 8. Knockdown of Dicer increases the Transwell invasiveness of Tca-8113 cells. Knocking down Dicer expression increased the ability of Tca-8113 cells to invade across a Matrigel membrane. More cells in the experimental group penetrated the membrane to the lower chamber than in the 2 control groups. (A) siRNA, Tca-8113 cells transfected with Dicer-siRNA; (B) mock, Tca-8113 cells transfected with negative siRNA; (C) Control, non-transfected Tca-8113 cells. Original magnification, x200.

Dicer is mis-expressed in different types of cancer, and Dicer expression changes are biomarkers of poor prognostics for cancer patients.

Cancer can be promoted or suppressed by miRNAs (19). In certain types of cancer, miRNAs are upregulated (20), but in most cancers the miRNAs are globally downregulated, and this downregulation plays a key role in the phenotypic transformation of cancer (4). Since Dicer is an upstream regulator of miRNAs, the mis-expression of Dicer in cancer can partly explain the abnormal expression of miRNAs in the cancer cells, and several studies have demonstrated changes in cancer behavior after silencing Dicer both in vivo and in vitro. For instance, knocking down Dicer in breast cancer can lead to a more malignant and invasive phenotype in vitro (21). Moreover, in animal studies, silencing Dicer in the mouse model of lung cancer using K-Ras promoted the development of the cancer, 

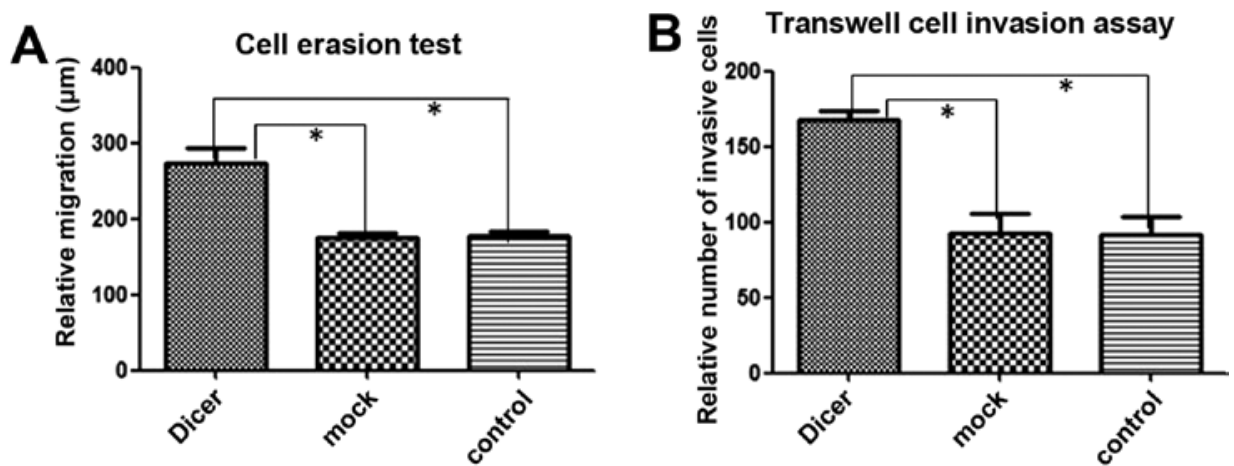

Figure 9. Silencing Dicer expression increases migration and invasion by Tca-8113 cells. Results of (A) the Erasion Trace test and (B) Transwell invasive assay showing enhanced migration and invasion by Dicer-depleted Tca-8113 cells. siRNA, Tca-8113 cells transfected with Dicer-siRNA; mock, Tca-8113 cells transfected with negative-control siRNA; control, non-transfected Tca-8113 cells; " $\mathrm{P}<0.05$.

and suppressing the expression of Dicer contributed to liver cancer in mice $(22,23)$. However, no evidence thus far has suggested a role of Dicer in tongue squamous cell carcinoma.

We found that Dicer was expressed at lower levels in 2 cell lines of tongue squamous cell carcinoma (Tca-8113 and UM-1 cells) compared to normal gingival epithelial cells, which supports the theory that miRNAs are downregulated in cancer due to the suppression of Dicer expression. Our results further link Dicer downregulation and the potential impairment of miRNA processing with the transformation of tumor cells; we also demonstrated that depletion of Dicer, a key component in the miRNA processing machinery in cells, enhanced malignant transformation of tongue squamous carcinoma cells. Specifically, knocking down Dicer in Tca-8113 cells significantly increased the proliferative ability, G1 arrest and invasiveness of the cells.

Collectively, our results suggest that Dicer functions as an indirect tumor suppressor, as silencing Dicer expression makes cancer cells more malignant. Although the mechanism of this transformation remains elusive, the present study suggests that rectifying the aberrant expression of Dicer could be one approach in treating tongue squamous cell carcinoma.

\section{Acknowledgements}

This study was supported by a grant from Guangdong Provincial International Science and Technology Cooperation Project (2012B050300028). The authors thank Dr AnXun Wang for providing the tongue squamous carcinoma cell lines (Tca-8113 and UM-1), and Professor Jun Xia from the Hong Kong University of Science and Technology for collaboration and guidance in the research.

\section{References}

1. Macrae IJ, Zhou K, Li F, et al: Structural basis for double-stranded RNA processing by Dicer. Science 211: 195-198, 2006.

2. Si ML, Zhu S, Wu H, et al: miR-21-mediated tumor growth. Oncogene 26: 2799-2803, 2007.

3. Shi B, Sepp-Lorenzino L, Prisco M, et al: MicroRNA-145 targets the insulin receptor substrate-1 and inhibits the growth of colon cancer cells. J Biol Chem 282: 32582-32590, 2007.

4. Lu J, Getz G, Miska EK, et al: MicroRNA expression profiles classify human cancers. Nature 435: 834-838, 2005.

5. Bu Y, Lu C, Bian C, et al: Knockdown of Dicer in MCF-7 human breast carcinoma cells results in G1 arrest and increased sensitivity to cisplatin. Oncol Rep 21: 13-17, 2009.
6. Han L, Zhang A, Zhou X, et al: Downregulation of Dicer enhances tumor cell proliferation and invasion. Int J Oncol 37: 299-305, 2010.

7. Tian-Run Liu, Li-Hua Xu, An-Kui Yang, et al: Decreased expression of SATB2: a novel independent prognostic marker of worse outcome in laryngeal carcinoma patients. PLoS One 7: e40704, 2012.

8. Lu Jiang, Yang Dai, Xiqiang Liu, et al: Identification and experimental validation of $\mathrm{G}$ protein alpha inhibiting activity polypeptide 2 (GNAI2) as a microRNA-138 target in tongue squamous cell carcinoma. Hum Genet 129: 189-197, 2011.

9. Nakayama S, Sasaki A, Mese H, Alcalde RE and Matsumura T: Establishment of high and low metastasis cell lines derived from a human tongue squamous cell carcinoma. Invasion Metastasis 18: 219-228, 1998-1999.

10. Chiosea S, Jelezcova E, Chandran U, et al: Up-regulation of Dicer, a component of the microRNA machinery, in prostate adenocarcinoma. Am J Pathol 169: 1812-1820, 2006.

11. Faber C, Horst D, Hlubek F, et al: Overexpression of Dicer predicts poor survival in colorectal cancer. Eur J Cancer 47: 1414-1419, 2011.

12. Papachristou DJ, Korpetinou A, Giannopoulou E, et al: Expression of the ribonucleases Drosha, Dicer, and Ago2 in colorectal cancinomas. Virchows Arch 459: 431-440, 2011.

13. Stratmann J, Wang CJ, Gnosa S, et al: Dicer and miRNA in relation to clinicopathological variables in colorectal cancer patients. BMC Cancer 11: 345, 2011.

14. Chiosea S, Jelezcova E, Chandran U, et al: Overexpression of Dicer in precursor lesions of lung adenocarcinoma. Cancer Res 67: 2345-2450, 2007.

15. Wu JF, Shen W, Liu NZ, et al: Down-regulation of Dicer in hepotocellular carcinoma. Med Oncol 28: 804-809, 2011.

16. Grelier G, Voirin N, Ay AS, et al: Prognostic value of Dicer expression in human breast cancers and association with the mesenchymal phenotype. Br J Cancer 101: 673-683, 2009.

17. Yan M, Huang HY, Wang T, et al: Dysregulated expression of dicer and drosha in breast cancer. Pathol Oncol Res 18: 343-348, 2012.

18. Pampalakis G, Diamandis EP, Katsaros D, et al: Down-regulation of dicer expression in ovarian cancer tissues. Clin Biochem 43: 324-327, 2010

19. Volinia S, Calin GA, Liu CG, et al: A microRNA expression signature of human solid tumors defines cancer gene targets. Proc Natl Acad Sci USA 103: 2257-2261, 2006.

20. Kumar MS, Lu J, Mercer KL, et al: Impaired microRNA processing enhances cellular transformation and tumorigenesis. Nat Genet 39: 673-677, 2007.

21. Martello G, Rosato A, Ferrari F, et al: A MicroRNA targeting dicer for metastasis control. Cell 141: 1195-1207, 2010.

22. Iwanaga $\mathrm{K}$, Yang $\mathrm{Y}$, Raso MG, et al: Pten inactivation accelerates oncogenic $K$-ras-initiated tumorigenesis in a mouse model of lung cancer. Cancer Ras 68: 1119-1127, 2008.

23. Sekine S, Ogawa R, Ito R, et al: Disruption of Dicerl induces dysregulated fetal gene expression and promotes hepatocarcinogenesis. Gastroenterology 136: 2304-2315, 2009. 